\title{
PREDICTIONS AND TESTS OF THE HYPOTHESIS OF LIMITING FRAGMENTATION *
}

\author{
J. C. VANDER VELDE \\ The University of Michigan, Ann Arbor, Michigan 48104, USA
}

Received 20 July 1970

\begin{abstract}
It is shown that the hypothesis of limiting fragmentation predicts the way in which the cross sections for production of fast particles in the laboratory should scale with beam energy. The agreement with experiment is investigated for the production of protons and pions in $p-p$ and $p-a$ luminum collisions in the beam momentum range 12 to $70 \mathrm{GeV} / \mathrm{c}$.
\end{abstract}

Present data on hadron-hadron interactions at c.m. energies above a few $\mathrm{GeV}$ show that about $80 \%$ of the cross section is inelastic and that the number of particles in the inelastic final states is increasing slowly with c.m. energy. It has been suggested by Benecke et al. that the gross (and perhaps even detailed) features of these multiparticle final states can be systematized by what they call the hypothesis of limiting fragmentation [1]. For the experimentalist this hypothesis (HLF) becomes a statement that the differential cross sections for particle production approach certain energy -independent limits as the beam energy becomes large.

In this note we show that a) HLF makes def inite and easily testable experimental predictions for fast particle production in the lab, b) present data are in remarkably good (although perhaps not exact) agreement with HLF, insofar as the shapes of the differential production cross sections are concerned, c) it is questionable whether the magnitudes of the cross sections obey HLF. In particular the empirical scaling law suggested by Liland and Pilkuhn [2] gives shapes which are consistent with HLF, but magnitudes which are definitely inconsistent.

Predictions of $H L F$. Consider the collision of two hadrons, $\mathrm{B}+\mathrm{T} \rightarrow \mathrm{B}^{\dagger}+\mathrm{T}^{\dagger}$, where $\mathrm{B}^{\dagger}$ and $\mathrm{T}^{\dagger}$ decay into one or more particles in the final state. (The possibility that $\mathrm{B}^{\dagger}=\mathrm{B}$ or $\mathrm{T}^{\dagger}=\mathrm{T}$ is supposed to be included here.) In the language of $\mathrm{HLF}, \mathrm{B}^{\dagger}$ and $\mathrm{T}^{\dagger}$ break up respectively into one or more fragments of $B$ (the beam particle) and $T$ (the target particle). One feature of HLF

\footnotetext{
* Research supported in part by the US Atomic Energy Commission.
}

says that in the rest frame of $T$, the differential cross section $\mathrm{d} \sigma / \mathrm{d}^{3} P$ for finding a given fragment of $T$ in a volume element $\mathrm{d}^{3} P$ around given values of $P_{\| 1}$ and $P_{1}$, is independent of the beam momentum $P_{0}$. (More precisely, the differential cross section approaches a limit as $P_{0}$ becomes large.) The variables $P_{\perp}$ and $P_{11}$ are cylindrical coordinates in momentum space along directions perpendicular and parallel to the velocity of $B$ with respect to $T$. A similar statement is made about the fragments of $B$ as measured in the rest frame of $B$. There are other features of HLF that involve the distributions of groups of fragments, but we will consider here only the single fragment predictions. It is implicit in HLF that measurements should be made in regions where the target fragments are not likely to be confused with the beam fragments. However, present data give us no clear indication of how to make this separation since there does not appear to be any depletion of pions or protons near $P_{1}^{*}=$ 0 in the c.m., at present beam energies. We will arbitrarily assume that the region in the lab $P_{\text {" }}$

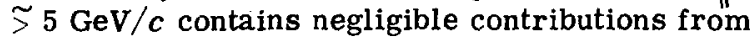
fragments of the target, and call this the 'fastlab' region. We will calculate the predictions of HLF for this fast-lab region which, by our assumption, contains only fragments of the beam particle $\ddagger$.

If we are looking at a particular fragment of the beam which is identical to the beam particle, then the additional difficulty arises that the case where the particle is one of several fragments of the beam cannot in general be distinguished from the case where the beam fragments into itself, leaving behind a fragmented target. For this type of data we are assuming that HLF applies to both cases so that the sum still obeys HLF. 
Let us imagine we are in the rest frame of $\mathrm{B}$ (primed coordinates) with $T$ coming in with velocity $\beta=P_{\mathrm{o}} / E_{\mathrm{O}}$ in the negative $P_{\|}$direction. ( $P_{\mathrm{O}}$ and $E_{\mathrm{O}}$ are the momentum and energy of $\mathrm{B}$ in the lab.) The projectile $\mathrm{T}$ causes $\mathrm{B}$ to fragment into a particular particle for which the differential cross section $\mathrm{d} \sigma / \mathrm{d}^{3} P_{\perp}^{\prime}=\left(2 \pi P_{\perp}^{\prime}\right)^{-1} \mathrm{~d} \sigma / \mathrm{d} P^{\prime} \mathrm{d} P_{\perp}^{\prime}$ does not depend on $\beta$, according to HLF. We now imagine two similar experiments done at two different values of $\beta$ (corresponding to two different values of $P_{0}$ ) which produce two identical fragments at a given $P_{11}^{\prime}$ and $P_{\perp}^{\prime}$ in the $\mathrm{B}$ rest frame. If we transform these two events into the lab frame, their coordinates are given by

$$
\begin{aligned}
& \left(P_{\perp}\right)_{1}=\left(P_{\perp}\right)_{2}=P_{\perp}^{\prime} \\
& \left(P_{\|}\right)_{1}=\gamma_{1}\left(P_{\|}^{\prime}+\beta_{1} E^{\prime}\right) \\
& \left(P_{\|}\right)_{2}=\gamma_{2}\left(P_{\|}^{\prime}+\beta_{2} E^{\prime}\right) .
\end{aligned}
$$

Therefore to a very good approximation (provided we avoid the region near $P_{\|}^{*}=0$ in the c. m., i. e., require $P_{\|}>\left(P_{\mathrm{O}} / 2 m_{\mathrm{T}}\right)^{1 / 2} m_{\text {fragment }}$ in the lab)

$$
\left.\left(P_{11}\right)_{2} / P_{11}\right)_{1} \approx \gamma_{2} / \gamma_{1} \approx P_{\mathrm{o} 2} / P_{\mathrm{o} 1}
$$

Likewise, the cross sections are related by

$\left(\frac{\mathrm{d} \sigma}{\mathrm{d} P_{\perp} \mathrm{d} P_{\mathrm{n}}}\right)_{2}=\left(\frac{E_{1}}{E_{2}}\right)\left(\frac{\mathrm{d} \sigma}{\mathrm{d} P_{\perp} \mathrm{d} P_{\mathrm{n}}}\right)_{1} \approx\left(\frac{P_{\mathrm{o} 1}}{P_{\mathrm{O} 2}}\right)\left(\frac{\mathrm{d} \sigma}{\mathrm{d} P_{\perp} \mathrm{d} P_{\mathrm{n}}}\right)_{1}$.

Eqs. (1) - (3) say that the quantity $P_{\mathrm{o}} \cdot \mathrm{do} / \mathrm{d}^{3} P$ is a function only of $P_{\perp}$ and $X=P_{\|} / P_{0}$, or that the density $\mathrm{d} \sigma / \mathrm{d}^{3} P$ at a given $P_{\perp}$ and $X$ is proportional to $1 / P_{0}$. In spherical coordinates this means

$\left(\frac{1}{P_{0}}\right) \frac{\mathrm{d} \sigma}{\mathrm{d} P \mathrm{~d} \Omega}=$ (function of $P_{\perp}$ and $\left.R=P / P_{\mathrm{o}}\right)$

where we have used the fact that $P_{\perp} \ll P_{\|}$so that $R \approx X$.

Measurements are usually made of $\mathrm{d} \sigma / \mathrm{d} P \mathrm{~d} \Omega$ at constant $\theta \approx P_{1} / P$, with varying $P$. Thus we can rewrite eq. (4), neglecting terms of order $\theta^{2}$,

$\left(\frac{1}{P_{\mathrm{O}}}\right) \frac{\mathrm{d} \sigma}{\mathrm{d} P \mathrm{~d} \Omega}=\left(\right.$ function of $\theta P_{\mathrm{o}}$ and $\left.R=P / P_{\mathrm{O}}\right)$.

Eq. (5) is precisely the scaling law discovered by Liland and Pilkuhn [2] except for the $1 / P_{0}$ factor on the left-hand side. Thus HLF predicts the way the variables scale in the LilandPilkuhn (L-P) law but the magnitude differs by a factor of $P_{0}$. For the data comparison made by $\mathrm{L}-\mathrm{P}$, the ratio of the predictions $\mathrm{HLF} / \mathrm{L}-\mathrm{P}=$
$70 / 19.2=3.64$, which is outside the quoted error in the $70 \mathrm{GeV} / c$ data of $\pm 50 \%[3]$.

We can summarize the predictions of HLF as follows. The fast-lab region, $P_{11} \sim 5 \mathrm{GeV} / c$, is populated by fragments of the beam and the density of events is proportional to $1 / P_{o}$ at a given value of $P_{\perp}$ and $X=P_{\|} / P_{0}$. Or in other words, $\left(2 \pi P_{1}\right)^{-1} \mathrm{~d} \sigma / \mathrm{d} P_{\perp} \mathrm{d} X$ is a function only of $X$ and $P_{\perp}$. In the c.m., as we increase $P_{0}$, the events simply get 'stretched out' in the $\pm P_{11}^{*}$ directions by an amount $\propto P_{\mathrm{O}}^{*} \approx\left(\frac{1}{2} M_{\mathrm{T}} P_{\mathrm{O}}\right)^{\sqrt[1]{ } / 2}$ so "that the density $\left(2 \pi P_{\perp}\right)^{-1} \mathrm{~d} \sigma / \mathrm{d} P_{\perp} \mathrm{d} P^{*}$ is proportional to $1 / P_{\mathrm{o}}^{*}$ at a given $X^{*}=P_{\|}^{*} / P_{0}^{*}$. "This makes

$\left(2 \pi P_{\perp}\right)^{-1} \mathrm{~d} \sigma / \mathrm{d} P_{\perp} \mathrm{d} X^{*}$ a function only of $X^{*}$ and $P_{\perp}$.

This behavior, predicted by HLF, agrees precisely with that predicted by Feynman [4] for what he calls an 'inclusive' measurement. We now turn to the question of whether present experimental data agree with the prediction.

Comparison with experiment. The HLF makes no predictions as to the variation of the cross section at a given $P_{\mathrm{o}}$ with respect to the variables $P_{\perp}$ and $P_{11}$. We must therefore compare data at different beam momenta $P_{0}$, using eqs.(3),(4), or (5), which refer to the fast-lab region. We can divide the question into two parts: a) Does the functional dependence on the variables (i.e., the shape) of the cross sections agree with the righthand side of (5)? b) Do the magnitudes of the cross sections agree with (5)? The second question is more difficult to answer at present since it appears to us that normalization uncertainties of as much as a factor of 1.5 can exist in any given experiment, and data from a single experiment in which $P_{0}$ was varied significantly are very scanty in kinematic regions where they can be compared.

Pion data. As we have already mentioned, the observations of Liland and Pilkuhn in comparing $\pi^{-}$production by protons on aluminum at 19.2 and $70 \mathrm{GeV} / \mathrm{c}$ are in very good agreement with HLF as far as the shape of the data is concerned. However, the magnitude of the $70 \mathrm{GeV} / c$ data is a factor of $\sim 3.5$ too small to agree with HLF. If the normalizations of the two experiments are correct then it is clear that HLF must be abandoned, or at least that it is not a useful concept at present energies. We discuss this point later in the article.

In fig. 1 we show data on production of fast $\pi^{ \pm}$ by protons [5]. The $30 \mathrm{GeV} / c$ data taken at $15 \mathrm{mrad}$ are compared with $19.2 \mathrm{GeV} / c$ data at $15(30 / 19.2)=23.4 \mathrm{mrad}$ (interpolated between 20 and $30 \mathrm{mrad}$ ). The agreement with $H L F$ is rather good, both in shape and in magnitude. We also show the $70 \mathrm{GeV} / c$ data for $\pi^{-}$produced by 


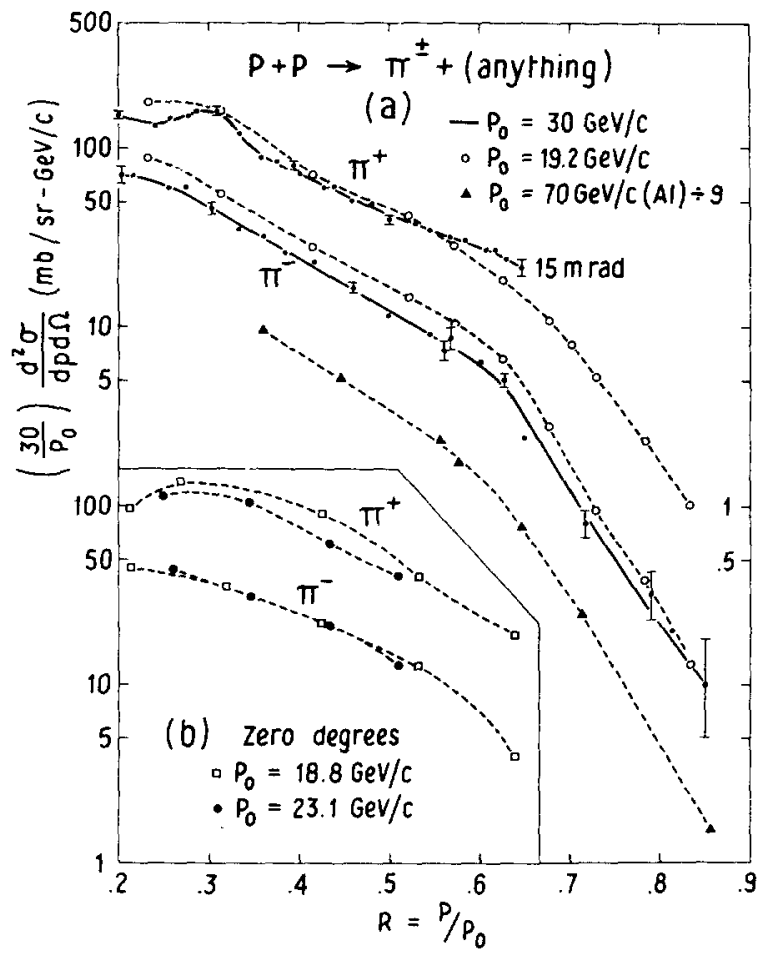

Fig. 1. a) $\pi^{+}$production compared at 19.2 and 30 $\mathrm{GeV} / c$, and $\pi^{-}$production compared at $19.2,30$, and $70 \mathrm{GeV} / c$. b) $\pi^{+}$and $\pi^{-}$production compared at 18.8 and $23.1 \mathrm{GeV} / c$. (Read lower left-hand scale.) The production angles quoted are the true angles times $\left(30 / P_{0}\right)$ in accordance with eq. (5) in text.

protons on aluminum, for $\theta=15(30 / 70)=$ $6.4 \mathrm{mrad}$ (interpolated from $0,6,12 \mathrm{mrad}$ data). We have divided the aluminum data by 9 to compare it to hydrogen + . In this case the agreement in shape is still good but the $70 \mathrm{GeV} / c$ data is too low by a factor of $\sim 3.5$. We also show on fig. 1 some zero degree $\pi \pm$ data taken in a single experiment at 18.8 and $23.1 \mathrm{GeV} / c$. Again the shapes agree but the magnitudes may be off, particularly for the $\pi^{+}$case. We have also compared data on $\pi \pm$ production by protons on $\mathrm{Be}$ from 12.5 to $30 \mathrm{GeV} / c$ [6] and find good agreement in shapes but are unable to reach any conclusions about magnitudes, possibly because of normalization uncertainties in the data.

We regard this agreement in shape, over several orders of magnitude, as rather striking confirmation of the variable-scaling predictions

+ Evidence for the correctness of this aluminum/hydrogen factor is discussed in ref. [2], but an uncertainty of $\pm 20 \%$ should probably be considered.

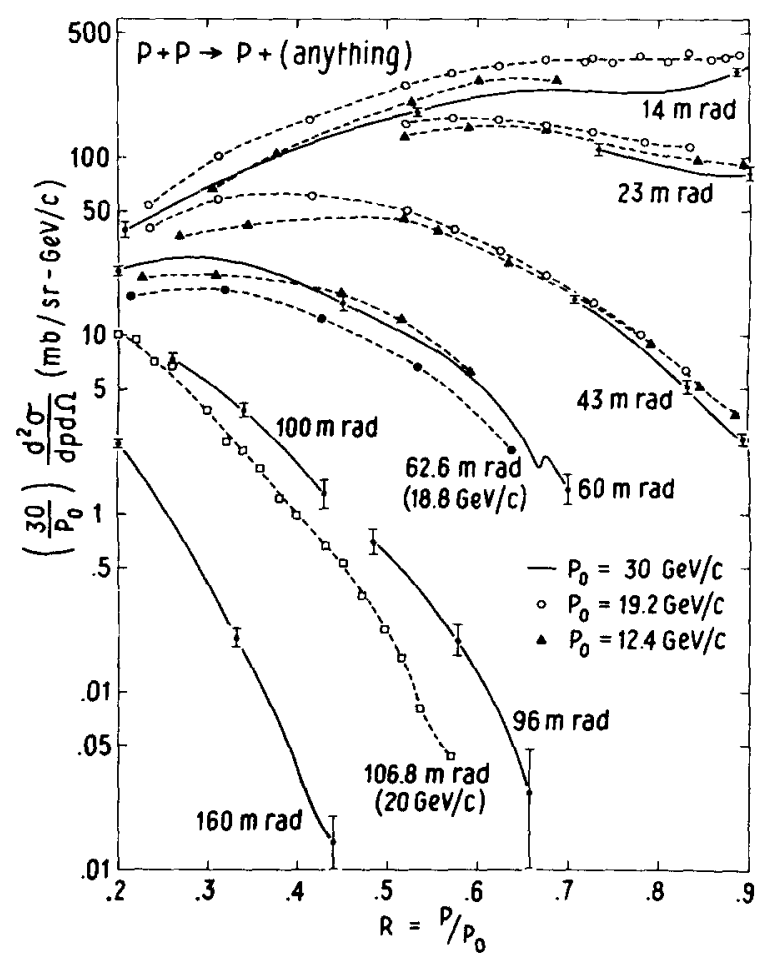

Fig. 2. Proton production compared at 12.4, 18.8, 19.2. 20 , and $30 \mathrm{GeV} / c$. The production angles have been scaled as in fig. 1. Some typical experimental errors are shown.

of HLF. For example, the $19.2 \mathrm{GeV} / c$ data disagree with the $30 \mathrm{GeV} / c$ data by as much as two orders of magnitude if we make the comparison in unscaled variables.

We mention further support for HLF pointed out by Yang [7] for the bubble chamber data of Smith et al. [8]. The integrated $P_{\perp}$ and $P_{11}^{*}$ distributions for observing $\pi \pm$ from various topologies appear to have approximately the correct $P_{\mathrm{o}}$ dependence. The $P_{\perp}$ distributions should be independent of $P_{0}$, and the $P_{\|}^{*}$ distributions, which the experimenters fit to $\alpha \cdot \exp \left(-\alpha P_{\|}^{*}\right)$, should give $\alpha \propto\left(P_{\mathrm{o}}\right)^{-1 / 2}$.

Proton data. In fig. 2 we show cross sections for fast-lab protons $(\geqslant 4 \mathrm{GeV} / \mathrm{c}$ ) produced in pp collisions at $12.4,18.8,19.2,20$, and $30 \mathrm{GeV} / c$ [9]. The 12.4 and $19.2 \mathrm{GeV} / c$ data were interpolated in angle. The 18.8 and $20 \mathrm{GeV} / c$ data were taken directly as published but are at slightly wrong angles. Again we regard the shape agreement as very impressive. The agreement in magnitudes is also reasonably good although not as conclusively in favor of HLF because of possible normalization uncertainties. There may 
also be some systematic variation with $\boldsymbol{P}_{\mathrm{o}}$ and angle.

Discussion. The agreement of present data with the variable-scaling prediction of HLF is very impressive as regards both pion and proton fragments of a proton beam particle. There may be some systematic deviations from the predicted shapes, but present data do not span the kinematic regions extensively enough to allow any conclusions of this type to be drawn. As noted by Benecke et al. [1], the cross section ratios $\mathrm{K}^{-} / \pi^{-}$and $\overline{\mathrm{p}} / \pi^{-}[3]$ are in rather good agreement with the variable-scaling prediction of HLF. It is important to note, however, that this does not test the magnitude-scaling, which is perhaps a more stringent test. It would be interesting to test the variable-scaling law using other beam particles and also to see whether the distribution of target fragments depends on the type of beam particle.

The agreement of present data with the magnitude of the cross sections predicted by HLF is, in our opinion, inconclusive. If the normalizations of the two experiments on $\pi^{-}$production from $\mathrm{A} 1$ at 19.2 and $70 \mathrm{GeV} / c$ are correct then the magnitude prediction of HLF is badly violated, since the data at $70 \mathrm{GeV} / c$ is a factor of $\sim 3.5$ too small along the line $P_{1} \sim 0$, and over a wide range of $P_{\|}$. Since the total cross section for making $\pi^{-}$is obviously not a factor of 3.5 smaller at 70 than at $19.2 \mathrm{GeV} / c$, this would mean that the $\pi^{-}$must be reappearing in other kinematic regions which have so far not been investigated. (There is no evidence for this at the few isolated wider angle points shnwn in ref.[2]). This is turn would mean that the shape predictions of HLF would have to be violated somewhere, which does not appear likely based on present data. We suspect, then, that the relative normalization of the 19.2 and $70 \mathrm{GeV} / c$ aluminum data may be off by a factor of $\sim 3.5$.

Whether HLF applies at present energies can be conclusively tested with present techniques by varying $P_{0}$ in a single experiment over as wide a range as possible, while scaling the experimental variables according to eq. (5).

I would like to thank Mr. John Cooper for his help in investigating and plotting the data, and Professors Henyey and Ross for some valuable comments.

\section{References}

[1] J. Benecke et al., Phys. Rev. 188 (1969) 2159.

[2] A. Liland and H. Pilkuhn, Phys. Letters 29B (1969) 663.

[3] Yu. B. Bushnin et al., Phys, Letters $29 B$ (1969) 48; F. Binon et al., Phys. Letters $30 \mathrm{~B}$ (1969) 506.

[4] R. P. Feynman, Phys. Rev. Letters 23 (1969) 1415.

[5] $30 \mathrm{GeV} / c$ p-p data: E. W. Anderson et al., Phys. Rev. Letters 19 (1967) 198;

$19.2 \mathrm{GeV} / c$ p-p data: $J$. V. Allaby et al., 14th Intern. Conf. on High energy physics, Vienna, 1968; $70 \mathrm{GeV} / c$ p-Al data: Ref. 3];

18.8 and $23.1 \mathrm{GeV} / \mathrm{c}$ p-p data: D. Dekkers et al., Phys. Rev. 137 (1965) B962.

[6] 18.8 and 23.1 p-Be data: Dekkers et al., ref. [5] $12.5 \mathrm{GeV} / \mathrm{p}-\mathrm{Be}$ data: R. A. Lundy et al., Phys. Rev, Letters 14 (1965) 504; 20 and $30 \mathrm{GeV} / c$ p-Be data: W. F. Baker et al., Phys. Rev. Letters 7 (1961) 101.

[7] C.N. Yang, talk given at the University of Michigan, 15 April 1970.

[8] D. B. Smith et al., Phys. Rev, Letters 23 (1969) 1064.

[9] The $12.4 \mathrm{GeV} / c$ data are from G.J. Marmer et al., Phys. Rev. Letters 23 (1969) 1469. The rest are from ref. [6]. 